\title{
LES ASSOLEMENTS FOURRAGERS EN REGIONS TROPICALES
}

par M.-G. CURASSON

De plus en plus on tend, en régions chaudes, à suivre l'exemple des pays tempérés où se généralise l'introduction, dans le système agricole, d'une sole fourragère sous la forme d'herbe récoltée et distribuée ou, au contraire, pâturée. Déjà complexe en Europe, la question l'est plus encore en pays tropicaux, à la fois parce que l'exécution se heurte à plus de difficultés, et parce que les buts recherchés par l'établissement du système sont plus divers.

Principes généraux. - Si, dans les pays tempérés, le système des assolements fourragers, bien qu'incomplètement étudié, est cependant largement appliqué, la durée des différentes soles variant avec la production principale, on est bien moins avancé en régions tropicales puisqu'on discute encore, pour certaines régions, du principe même de l'utilité du système, et que dans d'autres on en est encore à la période des essais. Avant d'examiner les conditions générales du procédé,'il faut d'abord le définir.

Dans les publications de langue anglaise, revient souvent l'expression leg. Si on emprunte à R. O. Whyte (1944) sa définition, le terme désigne surtout la culture, en prairie ou non, d'une ou plusieurs espèces fourragères, en rotation, à condition que ces espèces soient, pour le sol, améliorantes et non épuisantes. C'est donc une sole fourragère améliorante.

Une autre expression fréquemment utilisée est alternate husbandry. Le terme (culture alternée) s'entend de l'alternance, sur chaque champ d'une exploitation, d'une période de culture labourée (pour la production d'aliments à l'usage de l'homme ou des animaux, et de produits industriels) avec une période où le sol est utilisé par les animaux, soit comme pâturage, soit comme producteur de fourrage, la composition des espèces végétales étant conçue de façon à fournir aux animaux le maximum de fourrage qui convient à leur utilisation, et en même temps maintenir le sol en état de fertilité et de productivité pendant toute la durée du cycle de rotation (WhYte 1944). Traduite en français, l'expression est restrictive. On pourrait plus justement dire " assolements fourragers" ".

La " culture alternée » n'est pas un procédé qui peut être appliqué dans toutes les régions du monde. Cela dépend de l'état du sol, de la

Elev. et Méd. vét. des Pays tropicaux. - Arril-Juin 1948. 
possibilité d'y cultiver des plantęs fourragères, de créer et aménager des prairies, de la forme d'élevage pratiquée, des conditions économiques dans lesquelles la population agricole se tronve ou pent évoluer. Lé problème est d'autant plus varié que, dans les pays qui nous occupent, la diversité des asșociations agricoles possibles, selon les cultures principales d'une part et les soles fourragères d'autre part, la variété dès climats qui va d'une extrême sécheresse à une très forte humidité, ou encore d'une constance peu variable de la température à des oscillations thermiques très accentuéès; la variété des sols; tout cela intervient pour expliquer que beaucoup reste à étudier de ce point de vue. De toute façon, on peut considérer que " dans une combinaison donnée de sol et de climat, cette pratique est la meilleure qui puisse être adoptèe en vue du rendement optimum des cultures et des animaux, sans diminuer la fertilité du sol. Le système est très souple et peut souvent être adapté selon le type d'agriculture d'une région donnée. Il ne peut cependant être appliqué dans toutes les conditions et à tous les types d'agriculture n.

D'après JAcks (1944), unn des buts principaux du système est d'augmenter ou maintenir lâ fertilité du sol; cela est surtout dû à ce que; cette dernière étant particulièrement liée à l'état " granuleux " du sol — état qui s'oppose à la sécheresse et à l'érosion et favorise l'action des micro-organismes - il n'est pas de meilleure production, pour entretenir cette structüre, surtout dans les régions semi-arides, que la mise en herbe plus ou moins durable. Dans les régions plus arides, où les herbes poussent difficilement; ou dans les climats chauds et humides où la désagrégation du sol est facile, l'état granuleux est moins marqué.

Ce qui agit surtout dans cette "mise en herbe ", c'est le système radiculaire, qui intervient en divisant le sol et en lui' livrant de l'humus (et aussi, dans le cas des Légumineuses, par fixation de l'azote). Ia partie aérien'ne des hérbes fournit aussi de l'humus.

Des expériences poursuivies notamment dans les zones semi-désertiques de l'U. R.S.S., il résulte que, pour la fragmentation du sol, c'est un mélange de Graminées et de Légumineuses qui donne les meilleurs résultats, plutôt que les plantes d'une seule famille. De façon générale, les Graminées agissent én surface et les Légumineuses en profondeur. D'après KovalevskaJa (1940), l'augmentation de la perméabilité et de la porosité du - sol est surtout intéressante dans les régions arides où le sol est peu perméable en surface, en sorte que l'eau est évaporée avant d'être absorbée.

Il semble d'autre part (JACKs 1944) que l'effet complet d'une mise en herbe sur la structure du sol est obtenu en deux ans dans les régions humides, en quatre ans dans les régions sèches, et que la structure ainsi obtenue met un temps égal pour être détruite par la culture. 
Cépendant, on n'a pas encore fixé, pour les régions tropicales, les associations de Graminées et de Légumineuse qui pourraient être utilisées. On sait, en régions tempérées, ainsi que le fait observer Whyte (1944), que le succès des assolements fourragers dépend d'une rotation comprenant des soles de courte durée capables. de restaurer le sol pendant la période relativement courte au cours de laquelle il est occupé par l'herbe. L'amélioration apportée par la sole herbacée paraît, en régions tempérées, se maintenir pendant plusieurs années après que l'ncrbe a ćté labourée; mais en régions tropicales, la durée de cette amélioration, au lieu de se compter en années, pourrait se compter en mois. D'autre part, le taux de croissance et la production des herbes tropicales sont beaucoup plus importants que dans les climats froids, et ce développement rapide d'un système radiculaire important peut raccourcir le temps que met une culture herbacée à régénérer la structure du sol.

Il faut tenir compte aussi du fait que la conduite judicieuse des assolements fourragers suppose des exploitations agricoles bien aménagées et gérées, ce qui n'est pas le cas des fermes indigènes dont les propriétaires ont une éducation agricole et des moyens limités, alors que le milieu et le climat exigent, au contraire, des connaissances spéciales. Le problème est par ailleurs différent selon qu'on l'envisage en régions sèches ou en régions chaudes et humides.

Aux Etats-Unis d'Amérique, où la question de remise en herbe des régions semi-arides est importante, et où l'herbe peut précéder, dans la régénération de ces terres, d'autres cultures, les essais ont montré que si l'herbe diminue la production des cultures qui suivent la première année, cet effet ne dure pas; d'autre part, la seule amélioration de la structure du sol suffit à justifier l'introduction de l'herbe dans la rotation. Au point de vue économique, le procédé est également avantageux, à condition que la sole herbacée dure assez longtemps afin que soit combattu l'inconvénient que présente l'abaissement temporaire du rendement qui suit l'herbe.

En ce qui concerne plus particulièrement les régions tropicales humides, l'utilité des assolements fourragers tient à ce que, mềme là où on prend des mesures contre l'érosion, les cultures annuelles amènent. rapidement une diminution de la fertilité en raison de la décomposition rapide des matières organiques et du lessivage des éléments minéraux.

Les assolements visant à la restauration de la fertilité du sol peuvent se classer ainsi, en ce qui concerne les régions tropicales et semi-tropicales : $1^{\circ}$ la sole temporaire de Graminées et Légumineuses, comme en Nouvelle-Zèlande; $2^{\circ}$ d'autres cultures fourragères temporaires, plus résistantes à la sécheresse; $3^{\circ}$ les céréales cultivées pour la nourriture sur place; $4^{\circ}$ les engrais verts fourrages; $5^{\circ}$ la culture de Graminées des régions tropicales; $6^{\circ}$ la mise en jachère herbacée ou arbustive; 
$7^{\circ}$ la création dé pâturages de longue durée dans les régions semi-arides. Dans tous ces systèmes, sauf celui de l'engrais vert, intervient la mise au pâturage des animaux, selon des modes variés (N.chols, 1944).

Mais les assolements fourragers n'interviennent pas seulement dans les opérations visant à la restauration de la fertilité; ils ont leur part dans les systèmes agricoles où la production de plantes industrielles ou agricolès doit être intégrée dans une rotation qui combatte leur action épuisante.

Nous allons voir comment ccttc association a été réalisée en diverses régions:

Assolements fourragers divers. - La culture des Légumineuses fourragères, particulièrement comme plantes améliorantes, en assolements, a fait de gros progrès en régions tropicales au cours des récentes années, alors qu'elle élail auparavant peu pratiquée; on ne cultivait guère que des Légumineuses à destination spéciale et particulièrement pour l'alimentation de l'homme. Mais ainsi que le faisait remarquer Chevalier (1924), on vit peu à peu la nécessité des engrais verts et surtout de la culture des Légumineuses en rotation triennale et de l'emploi du fumier de ferme; les engrais verts ne rendent pas toujours Ies mêmes services qu'en régions tempérées, particulièrement là où les termites les détruisent avant qu'ils ne soient décomposés. Pour cela, et aussi parce qu'il faut peut-être préférer la solution qui consiste à nourrir avec ces Légumineuses des animaux qui donnent un fumier riche et rapidement assimilable, il semble préférable de cultiver des Légumineuses fourragères en rotation triennale avec toutes les plantes un peu épuisantes.

Cette question des Légumineuses fourragères tropicales est loin d'être au point. C'est que les espèces locales sont souvent rares ou ne répondent pas aù but cherché. Ọn est alors tenté d'importer des espèces qui ont réussi ailleurs; mais ces introductions ont souvent causé des déboires. En général, ainsi que le fait remarquer Van Rensburg (1947), il y a fréquemment, au début, un certain enthousiasme qui cède trop souvent à l'expérience; plus encore que les Graminées, les Légumineuses ne réussissent que si les conditions qu'elles trouvent sont celles deleur milieu originel. Les petites espèces, en particulier : Trifolium, Desmodium, Stylosanthes, se montrent souvent assez vigoureuses au début, puis cèdent peu à peu la place, surtout dans les régions semi-arides, aux Graminées én touffes.

L'usage des'assolements est préparé en régions tropicales par le fait que, les plantes cultivées par l'homme primitif servanten général à tous ses besoins, on trouve peu d'exemples de monoculture; quand l'agriculture évolue, on continue le système des cultures mixtes, puis on arrive aux assolements, le plus simple étant la succession de la jachère 
au champ cultivé; il en est de bien plus compliqués, la complexité venant de la variété des cultures, du rythme des rotations, etc. La succession des cultures n'est pas forcément annuelle; dans de nombreuses régions, plusieurs récoltes se succèdent sur le même sol pendant la même année; c'est ainsi que, dans l'Irak, en deux années, on cultive le cotonnier de mars à octobre; une céréale de novembre à mai; des Légumineuses de juin à mars. En Nigéria, l'assolement devient très compliqué; on trouve la succession suivante:

1. Maïs .................... de Septembre à Février.

2. Ignames semés dans le rnaïs........ de Novembre à Septembre.

3. Maïs semé entre les lignes d'ignames de Mars à Aolit. Cotonnier entre le maïs et les ignames. d'Août à Février.

4. Maïs ................ Mars à Aoùt.

5. Manioc semé dans le maïs ......... de Mai à Janvier.

6. Puis jachère pendant plusicurs années.

Dans certaines parties de l'Afrique, on a cherché un assolement pour les régions où l'élevage est impossible, mais où les Léguminenses sont nécessaires pour conserver la fertilité des terres. Sur quatre ans, on a suivi l'assolement suivant :

1. Stizolobium suivi de maïs.

2. Soja suivi de tabac ou de cotonnier.

3. Stizolobium suivi de patates.

4. Maïs suivi de Vigna sinensis.

On remarquera que cet assolement peut convenir à une région d'élevage.

On cultive souvent les Légumineuses en même temps que les Graminées, ces dernières servant la plupart du temps de support. Le choix de ces associations varie évidement avec le terrain, le climat, la vitesse de végétation des plantes, etc. Dans les pays subtropicaux, l'association vesce-avoine est préférée; dans les régions plus chaudes, on peut employer le maïs comme support et comme Légumineuse, des Vigna.

La valeur alimentaire moyenne de diverses associations est la suivante (VELU) :

\begin{tabular}{|c|c|c|c|c|c|c|}
\hline & Eau & Cend. & Mat.all & Cellulose & H. de C. & Mat.gr. \\
\hline$\pi$ & 80 & & $a_{7}$ & - & & \\
\hline Maïs et cow pea. & 80 & 1,8 & 2,1 & 5,3 & 10,4 & 0,4 \\
\hline Avoine et cow pea ..... & 78,2 & 2,2 & 4.5 & 5.7 & 8.5 & 0,9 \\
\hline Avoine et pois fourrager. & 77,4 & 2,0 & 3,2 & 6,3 & 10,1 & 1,0 \\
\hline Orge et vesce & 80 & 1,2 & $\stackrel{2}{\sim} 8$ & $6, \overline{0}$ & 9 & 0,5 \\
\hline Avöine et vesce... & 77,3 & 1,6 & 3,3 & 7,1 & 10,1 & 0,6 \\
\hline Sorgho et cow pea. . . . & 81,3 & 1,7 & 1,5 & $5, \overline{0}$ & $9, \overline{0}$ & 0,5 \\
\hline Kafir et soja . . . . . . . & 82,9 & 2,1 & 2 & 6,2 & 6,2 & 0,6 \\
\hline
\end{tabular}


Voici, à titre d'indication, ce que donnent en moyenne diverses Légumineuses dans la vallée du Niger (par hectare et en sec).

\begin{tabular}{|c|c|}
\hline Velvet bean ............. & 6 à 8 tonne \\
\hline Arachide...$\ldots \ldots \ldots \ldots$ & 3 à $5-$ \\
\hline Crotalaria juncea......... & 5 à $6, \quad-$ \\
\hline Niebé (Vigna sinensis). & 5 à 8 \\
\hline
\end{tabular}

En vert, le Crotalaria juncea peut donner 25 tonnes à l'hectare.

Dans les Indes occidentales, on cultive comme fourrages et plantes de couverture, diverses Légumineuses parmi lesquelles dominent: $\mathrm{Mu}$ cuna aterrima, Dolichos labblab, Vigna unguiculata, Phaseolus aureus, Soja hispida; Phaseolus mungo, Cajanus cajan; mais toutes ces plantes à vie courte ne peuvent entrer dans le cycle de rotation dans le but de maintenir la fertilité du sol. Il faut pour cela une Légumineuse vivace tenant le sol assez longtemps. Mieux que toute autre, la luzerne remplit. ce but en pays tempéré. Elle réussit aussi en certaines régions chaudes, mais sous irrigation. Ailleurs, on n'a pas encore trouvé de Légumineuse pouvánt la remplacer de façon certaine.

A la Trinité (Paterson 1944), on obtient une bonne couverture avec les plantes suivantes, plus ou moins durables : Cientrosema pubescens, Calopogonium mucunoïdes, Dolichos hosei, Indigofera endecaphylla, Pueraria javanica. Toutes sont lentes à se développer et demandent beaucoup d'entretien pour éviter leur envahissement par les mauvaises herbes. La meilleure de ces Légumineuses est Indigofera endecaphylla; à Porto-Rico, on l'utilise maintenant pour lutter contre l'érosion et maintenir la fertilité du sol. Cette plante, originaire de l'Inde, a été introduite aussi de Ceylan aux îles. Hawaï; elle couvre rapidement le terrain, est résistante à la sécheresse et au pacage. On y a recours aussi en Indo-Chine. Stylosanthes guyanensis est aussi une plante qui, dans les mêmes conditions, peut servir à la fois d'engrais vert et de fourrage. D'autres espèces du genre sont recommandées comme succédanés de la luzerne: C'est le cas pour.St. mucronata, la "luzerne sauvage" du Queensland, St. montevidensis, d'Amérique du Sud, St. viscosa, d'Afrique occidentale.

Voici maintenant les principales Légumineuses fourragères qui peuvent entrer en assolement.

L'arachide-fourrage entre en rotation avec le coton, avec des Graminées; on l'associe aussi à ces dernières, notamment avec le maïs.

Le pois d'Angolè, Cajanus indicus, souvent cultivé comme arbuste d'ombrage pour certaines cultures, peut ètre utilisé comme plante améliorante; il tient le sol plusieurs années sans grande façon culturale; mais il lui faut un sous-sol humide. On donne les feuilles en vert, après la récolte des graines; ou bien on coupe les branches plusieurs fois par an, avant maturité des gousses; ou encore, on coupe au ras du sol au 
fur et à mesure de la formation des branches; on évite ainsi une lignification rapide. On a pu obtenir ainsi 60 tonnes à l'hectare; la farine de feuilles vaut la farine de luzerne. On peut aussi ensiler. Calopogonium mucunoüdes est une espèce sud-américaine qu'on recommande dans les plantations de café pour la fixatıon du sol. Sa valeur est un peu inférieure à celle de la luzerne. En Côte d'Ivoire, on lui a reproché de se dessécher après la saison des pluies, ce qui peut faire courir des risques d'incendie.

Centrosema pubescens est utilisée dans les Indes occidentales comme plante de couverture, et dans les zones arides d'Argentine comme fourrage. Dans les zones humides d'Afrique occidentale (Gold Coast), elle pousse abondamment et peut nourrir plus d'un mouton par 4 ares.

Crolalaria juncea, qui est susceptible d'adaptation dans bien des pays tropicaux et subtropicaux, entre souvent en rotation avec le riz; on la donne surtout en vert. On cultive aussi comme engrais vert et accessoirement comme fourrage diverses autres espèces du genre, dans les planiations de café, de tabac. On sait que plusieurs espèces sont dangereuses : $C$. speclabilis, d'Amérique, $C$. dura, $C$. burkeana, d'Afrique du Sud, C. mitchellii, d'Australie, C. mesopontica, d'Afrique orientale. Ciela doit rendre circonspect quand on tente l'introduction d'une variété nouvelle dans une région.

Cyamopsis psoraloïdes ( $=C$. letragonolocus) est cultivée dans l'Inde comme engrais vert, et aussi aux Etats-Unis. Au Soudan français, elle s'est montrée un bon fourrage vert; comme foin, elle est moins bien appétée. On l'accuse à tort d'ètre toxique; de même pour $C$. senegalensis, bonne espèce qui, au Sénégal, reste longtemps verte et est très prisée des bœufs (indigo du bœuf). Cette espèce, comme $C$. slenophylla, de la zone sahélienne, mériterait d'être étudiée.

Parmi les diverses espèces de Desmodium (= Meibonia) qui sont utilisées comme plantes de couverture, ou comme plantes améliorantes, beaucoup sont des fourrages médiocres, en raison de leur haute teneur en cellulose. Quelques-unes fournissent cependant un appointimportant. D. lorluosum est une espèce d'Amérique du Nord qui, dans l'Inde, atteint 2 à 3 mètres, fournit un fourrage ligneux ( $50 \%$ de cellulose), auquel les animaux s'habituent cependant, surtout si on coupe jeune. Une espèce voisine est $D$. maurilianum, qui est spontanée dans tous les pays tropicaux et est recherchèe bien que ligneuse. Le " trèfle espagnol ", $D$. uncinalum a un système radiculaire qui lui permet d'être résistant à la sécheresse ; il s'étend très rapidement. Une espèce voisine D. canum, plus petite, résiste bien à la pàture, mais demande de l'humidité. Aux Indes occidentales on cultive $D$. virgalus qui sert à fabriquer une farine analogue à la farine de luzerne. De nombreuses espèces d'Amérique du Sud, particulièrement des zones de savane, ont excellente réputation. $D$. discolor en particulier vaudrait au Brésil toutes les légumineuses d'Europe, y compris la luzerne. Citons aussi une 
espèce australienne, $D$. triflorum qui a été introduite aux îles Hawaï, et qui peut vivre dans les sols les plus ingrats.

Les Doliques; cultivés pour leurs graines ou comme fourrages entrent dans divers assolements. L'espèce la plus connue est Dolichos labblab qui a été introduite de l'Inde dans diverses régions tropicales. Il en existe diverses variétés, grimpantes comme le haricot commun, ou couchées sur le sol. Au Soudan français, introduite dans le cycle de la culture du coton, la plante s'est révélée un bon fourrage. Dolichos biflorus, également de l'Inde, y est cultivée pour sa graine et comme fourrage. On l'a cultivée au.Sénégal en 1943; la plante s'est montrée peu exigeante et paraîtrait mériter d'être introduite en zone sablonneuse. D. uniflorus, qui serait une forme de l'espèce précédente, en a les qualités. Citons aussi : $D$. hosei utilisée en Indochine comme plante de couverture et que le bétail mange volontiers (aux Indes occidentales également). D. bulbosus (= Pachyrhizus angulatus) recherchée par le bétail en Nouvelle-Calédonie et dont la racine tubéreuse est distribuée aux'vaches aux Antilles (pois-cochon).

Glycine hispida = Soja hispida entre dans divers assolements. Le soja est trop connu pour que nous nous y arrêtions ici. Une espèce voisine, Glycine javanica, vivace, a été employée utilement comme agent de conservation du sol en Afrique du Sud et au Tanganyika. C'est un bon fourrage:

Nous avons $\forall u$ qu'on utilise comme engrais vert et fourrage, en diverses régions tropicales, Indigofera endecaphylla. $\mathrm{D}^{\top}$ autres espèces du genre sont intéressantes : en Indochine; I. teysmannii qui contient jusqu'à $32 \%$ de matières azotées; en Afrique orientale, I. arrecta, $I$. retroflexa, I. sunvenlens; dans les régions arides d'Amérique du Sud, I. lespedezoïdes, I. pascuorum.

Dans le genre Lalhyrus, les espèces ne peuvent guère prospérer qu'en régions subtropicales. I.e pois de Tanger; Lathyrus tingitanus, s est étendu én Amérique, en Australie comme engrais vert, plante de couverture ou fourragé.

Les Lespedeza sont des Tégumineuses-Hédysarées qui habitent pour la plupart l'Asie et l'Australie. Plusieurs espèces cultivées dans les zones chaudes d'Amérique permettent d'utiliser des sols acides et pauvres et de s'opposer à l'érosion. La principale est $L$. sericea, assez bon fourrage, mais pauvre en phosphore.

Le genre Leucaena parmi une vingtaine d'espèces américaines à grains comestibles (dont $I$. esculenta, L. pulverulenta) comprend $L$. glauca, originaire probablement de l'Amérique centrale, des Antilles, et qui a été répandue dans toutes les régions chaudes du globe. Cet arbuste est utilisé comme plante de couverture, engrais vert et fourrage. A l'ìle Maurice, la plante a acquis la réputation de provoquer à la longue de l'alopécie chez les chevaux; cela n'a pu être vérifié dans l'Inde où, pàr 
contre, on aurait observé que l'urine contracte une odeur acide qui se transmet aussi au lait. $L$. glauca est largement cultivée aux Indes occidentales; on en fait une farine ressemblant à la farine de luzerne et renfermant $13 \%$ de protéines.

Plusieurs espèces du genre Medicago sont des plantes fourragères. La , première place revient à la luzerne, $M$. saliva, qui a envahi les régions subtropicales et certaines régions tropicales où est possible l'irrigation. On a essayé d'acclimater des espèces plus résistantes à la sécheresse : $M$. arborea spontanée en Afrique du Nord (luzerne arborescente) ne paraît pas devoir s'étendre en régions tropicales; un essai au Soudan français a échoué; de nombreuses variétés de cette espèce peuvent être intéressantes en régions subtropicales; elles sont feuillues et précoces. $M$. hispida, M. falcala, M. denliculala, $M$. minima, ne paraissent pas non plus devoir quitter les régions subtropicales.

Si le Melilot ordinaire ne peut affronter les régions tropicales, on peut, par contre, utiliser des espèces voisines dans les régions chaudes, comme plantes améliorantes et fourrage. Dans l'Inde, on cultive deux espèces voisines, $M$. ilalica et $M$. parviflora, qui réussissent môme dans la zone tropicale. Dans le Punjab, on cultive $\boldsymbol{M}$. parviflora sous irrigation.

Deux genres voisins. Mucuna (=Desmodium) et Stizolobium, souvent confondus, offrent des espèces intéressantes. Slizolobium utile, Mucuna utilis, $M$. artropurpurea, le pois noir, pois mascote, est cultivè à Maurice, à la Trinité, en Tasmanie, comme plante d'assolement, comme engrais vert dans la culture de la canne à sucre, et aussi comme fourrage riche en protéine.

Plusieurs espèces sont cultivées sous le nom de velvet bean (haricot velouté), surtout en Amérique, mais aussi dans diverses régions tropicales, comme plantes améliorantes, plantes de couverture ou fourrage. On choisit celles qui ne sont pas trop velues, pour ne pas être dangereuses. C'est le cas pour $S$. utile, $S$. nivea. En Amérique tropicale, on cultive le maïs comme support, et on fait pâturer cette associalion après récolte des é pis et des gousses à la main. Dans les régıons où on cultive le coton, les velvet bean en sont les compagnons ordinaires avec le maïs; $\mathrm{Au}$ lieu de semer sur la même raie que le maïs, on peut alterner les raies; certaines variètés buissonnantes se passent de support. Parmi les autres espèces, citons St. cochinchinensis (= Mucuna cochinchinensis, Marcanthus cochinchinensis) qui serait une espèce très méritante; St. pruriens (= Mucuna edulis) cultivé dans l'Inde, à la saison des pluies, surtout en climat subtropical; St. deeringianum, espèces maintenant cultivées dans beaucoup de régions chaudes, surtout d'Amérique du Sud, où l'on emploie comme plante de couverłure dans les plantations de café (Brésil) et les terrains sablonneux de Cuba. Elle pourrait réussir en zone soudanaise.

La sensitive, Mimosa pudica, répandue dans diverses régions tropicales 
comme engrais vert et plante de couverture, est considérée parfois comme un bon fourrage. Les animaux mettent un certain temps à s'habituer à la pâture, en raison des poils piquants. Plusieurs espèces voisines des zones arides et chaudes d'Amérique du Sud ne manquent pas d'intérêt, $M$. cabrera, M. coesalpinifolia, M. vernicosa, etc. La u sensitive de Montravel ", Desmanthus virgalus, serait aussi un bon fourrage (Nouvelle-Calédonie).

Les diverses espèces du genre Phaseolus qui sont cultivées comme fourrages entrent exceptionnellement en assolement.

Pueraria thunbergiana, le $K u d z u$, originaire d'Asie, a été introduit en Afrique, en Amérique. Le Kudzu s'adapte à des conditions très variées. En Afrique du Sud, on a pu l'utiliser à l'amélioration de terrains très pauvres où aucune autre légumineuse ne pouvait pousser. Sa racine très grosse lui permet de résister aux grandes sécheresses; il croît avec exubérance, s'étend rapidement dans toutes les directions tt constitue ainsi un excellent agent contre l'érosion. G'est un bon aliment du bétail, mais on recommande en général de n'en pas donner abusivement. Sous ce nom de Kudzu, on cultive aussi en Afrique du Sud $P$. hirsute: $P$. javanica est une plante de couverture utilisée également comme fourrage aux Indes occidentales. On y a eu recours dans les plantations de palmiers à huile en Afrique occidentale.

Sesbania ægypliaca est cultivée dans l'Inde, surtout pour ỉes moutons et les chèvres, parfois en assolement avec la canne à sucre; on y a recours aussi comme haie, comme brise-vent; on taille à hauteur voulue el on donne les émondes au bétail. On considère la plaule comme plus fácile à obtenir que la luzerne, résistante, et pouvant, si l'eau est abondante, donner plus de 300 quintaux de fourrage vert à l'hectare pendant 3 ans. ' $S$. 'xgyptiaca existe aussi en Afrique occidentale, ainsi que $S$. aculeata, S. punctala, qui pourraient aussi servir aux mêmes fins. Outre S. xgypliaca, on cultive dans l'Inde $S$. grandiflora, dont les feuilles sont mangées par l'homme comme par les animaux. Les deux espèces peuvent être cultivées en rotation avec le coton. On sait que plúsieurs espéces américaines sont dangereuses.

On peut introduire dans les rotations' plusicurs Stylosanthes; St. guyanensis et St. guyanensis var. subviscosus sont des substituts de la luzerne moins exigeants quant à l'eau. St. viscosa est une espèce d'Afrique occidentale qui serait à essayer. St. mucronata, originaire des Antilles et de l'Amérique tropicale, a été naturalisée en Asie tropicale. Elle vaudrait la luzerne.

Parmi les nombreuses espèces non toxiques du genre Tephrosia, il est des espèces africaines qui peuvent être utilisées comme engrais vert et comme fourrage. Leurs qualités mériteraient d'êtres étudiées. Citóns', en Afrique occidentale, $T$. ansellii, $T$. linearis, $T$. bracteolata, T. teptostachya; en Afrique orientale, T. incarna, $T$. purpurea. 
Les trèfles des régions tempérées vivent mal ou donnent de faibles rendements en régions chaudes. Dans les pays subtempérés chauds, le trèfle d'Alexandrie réussit. On le cultive en Égypte, en Afrique du Nord, dans l'Inde septentrionale, en Anérique du Sud. Dans l'Inde, on le cultive ordinairement en rotation avec les crucifères (Brassica, Sinapis). $T r$. fragiferum est intéressant en raison de son aptitude à pousser en terrains salés ou alcalins; il est utilisé en Argentine, en Rhodésie, en Australie.

Une espèce spontanée en Afrique du Nord (et même dans le Midi de la France), en Asie occidentale. Tr. resupinatum, entre en rotation dans l'Inde (shaflal), en Afrique orientale et occidentale ; Tr. ruppelianum (=subrolundum) serait une des rares espèces de trèfle qui puisse s'accommoder du climat tropical et pourrait être cultivée en Afrique occidentale en saison des pluies ou sous irrigation, d'après A. Chevalier. Tr. sublerraneum a penmis d'utiliser en Australie de vastes étenducs de terrain naguère incultes; des accidents lui sont dus, qui tiennent à l'existence d'une substance œstrogène que contiendraient certaines variétés.

Les diverses Vesces, cultivées seules ou avec une graminée-support, ne conviennent qu'aux régions subtropicales.

Pàrmi une vingtaine d'espèces du genre Vigna domine Vigna sinensis (=V.catjang), le cowpea, dolique mangette, niébé, etc., qui a gagné à peu près toutes les régions tropicales, seul ou en association avec d'autres fourrages. C'est vraisemblablement une des légumineuses les moins exigeantes au point de vue du terrain (surtout sabonneux). Il existe de nombreuses variétés adaptées à diverses régions sablonneuses sèches. Dans les régions où, en Amérique, on cultive le coton, on sème les cowpeas en raies alternées avec le maïs, comme on le fait avec les velvet beans, et on fait pâturer quand le maïs est récolté. Les cowpeas sont aussi cultivés seuls, en diverses rotations, et constituent alors un excellent fourrage. On cultive avec le Soudan grass, les sorghos, le Johnson grass comme supports.

Vigna maritima (=Vigna lutea, Phaseolus marilimus) est ane plante de couverture utilisée en diverses régions, particulièrement aux Philipines, dans les plantations de palmiers et d'hévéas. C'est un excellent fourrage.

Vigna unguiculala, dans l'Inde, à Maurice, au Kenya, est cultivée comme plante améliorante, comme engrais vert, comme fourrage, en mélange avec le sorgho, le maïs; tous les terrains lui conviendraient, sauf les terrains argileux lourds. Deux espèces africaines, $V$. lexillata (qu'on trouve aussi en Amérique du Sud) et $V$. nilotica, mériteraient d'être étudiées.

Parmi les rares légumineuses spontanées qui, dans les zones sablonneux de la zone sahélienne de l'Afrique, pourraient être introduites 
dan's diverses rotations; Zornia diphylla serait intéressante, comme Alysicarpus vaginales. On la retrouve en d'autré régions africaines, à Madagascar, en Argentine.

Nous allons maintenant examiner quelques types d'assolements dans la culture du coton, du riz, de la canne à sucre.

Colon.- En ce qui concerne particulièrement la culture du coton, à laquelle l'élevage est souvent associé, cette culture, épuisante, ne se fait pas sans fumure, accompagnée de rotation, de mise en jachère, etc. Dans l'Inde, on a couramment recours à ces rotations : le Sorghum vulgare, le mails, Cajanus indicus. Les légumineuses de couverture jouent un rôle important. En Égypte, la fertilité est maintenue par la culture du bersim, Trifolium alexandrinum. Dans l'Inde, on utilișe la même plante, et aussi le Trifolium resupinalum, le Melilolus parviflora. Ces fourrages sont utilisés pour les bêtes de travail, les laitières, au moment où sans elles il n'y. aurait guère que du fourrage sec.

Malheureusement ces cultures fourragères demandent beaucoup d'eau, et ne peuvent être pratiquées que sous irrigation abondante.

Au Soudan français, dans la culture sèche du coton, on a adopté l'assolement triennal suivant:

$$
\begin{aligned}
& \text { Coton } \ldots \ldots \ldots \ldots \ldots \ldots \ldots \\
& \text { Manioc } \ldots \ldots \ldots \ldots \ldots \ldots \ldots \\
& \text { Arachide } \ldots \ldots \ldots \ldots \ldots \ldots \text { Pois d'Angole. } \\
& \text { Sorgho ou Maïs. }
\end{aligned}
$$
lablab:

On peut aussi utiliser les velvet beans, Vigna sinensis, le dolique

En culture sèche, la fertilité du sol pourrait être maintenue par un assolement triennal comportant en tête une sole de coton fumée à 10 tonnes à l'hectare; en deuxième année, une culture vivrière, et en troisième année, une légumineuse (arachide). Mais, l'indigène n'arrivant pas à prodıire la quantité de fumier suffisante, on doit adopter un assolement plus extensif, permettant un roulement'de culture et de jachère qui assure le repos des terres.

Fin culture irriguée, le même assolement triennal donne de bons résultats, mais il n'est pas assez intensif.

Dans le Queensland, où les fermes à coton élèvent du bétail laitier, on obtient de bons résultats en pratiquant une rotation qui comprend 3 années de coton, puis 3 années de Chloris gayana; aux États-Unis, le coton entre on rotation avec un mélange trèfle incarnat-Lolium ou Lespedeza-avoine. On a aussi préconisé, au Queensland, un système qui comprendrait 3 ou 4 annẻes de culture du coton; puis des céréales pâtưrées ou fanées et du sorgho (pour les porcs) et enfin une période de Rhodes grass, qui peut subsister 10 ans.

Quand le sol a été épuisé par le coton, en Ouganda", on préfère, pour 
la régénération, les Graminées aux Légumineuses, les meilleures étant des Brachiaria ou Penniselum purpureum; mais le sol peut être trop pauvre pour permettre d'emblée l'installation de ces herbes; dans ce cas, on commence avec Rhynchelylrum repens qui prépare le terrain aux autres Graminées.

D'autre part, les expériences faites en U.R.S.S. dans les régions où l'on cultive le coton ont montré que la luzerne est de beaucoup la meilleure culture qui, sous irrigation, doit précéder le coton (et aussi le riz).

Riz. - Dans le cas spécial de la culture du riz, l'intervention du bétail varie; en certaines régions, on adopte le système qui comprend une année de culture du riz et deux années de pâturage. Le bétail pâture les chaumes du riz, utilise la paille pendant l'hiver et des plantes fourragères succèdent au riz. Cependant divers auteurs considèrent que la riziculture, sous les tropiques, peut, demeurer une monoculture pendant de nombreuses années successives.

Dans l'Inde, les buffles de travail sont nourris de paille de riz et d'herbes ramassées çà et là; aussi ces animaux sont en assez médiocre état. Dans les terres irriguées au moins, on peut faire une culture dérobée de légumineuses après le riz. Dans beaucoup de régions telles que le Sund et le Mysore, on cultive de grosses quantités de sorgho, mais on y adjoint aussi des légumineuses pour améliorer les rations nutritives des buffles. Le Bersim est particulièrement intéressant; la luzerne également.

Dans l'Inde (ferme de Pusa), on a obtenu de bons résultats dans la production du fourrage pour les vaches laitières, en utilisant la rotation suivante :

\begin{tabular}{l} 
Première récolte (saison chaude) \\
$1^{\text {re }}$ année \\
\hline
\end{tabular}

Maïs pour ensilage Maïs pour la graine Légumineuses vertes et fourrage

DEuXİ̀me RÉCOLTE (saison froide)

$\begin{array}{lll}1^{\text {re }} \text { année } & 2^{\text {e année }} & 3^{e} \text { année } \\ \text { Avoine } & \text { Cajanus indicus } & \text { Avoine }\end{array}$

Un certain nombre de plantes peuvent remplacer le maïs dans la rotation : l'Andropogon sorghum qui donne du fourrage vert, mais dont l'ensilage rẻussit moins bien que celui du maïs; certains bambous comme le Dendrocalamus strictus; quelques variétés de canne à sucre, l'herbe"de Guinée, le Rhodes grass.

La culture de l'arachide sur le même terrain arrive assez vite à l'épuiser; c'est ce qu'on observe au Sénégal; en Floride, où on a voulu 
éviter lés inconvénients de la monoculturé, Spencer et Brown ont recommandè l'assolement sujvant:

1. Coton et Covercrop (couverture) d'hiver;

2. Maïs et Velvet bean (Stizolobium);

3. Arachide;

4. Avoine ou Cowpea (Vigna sinensis);

ou bien:

1. Arachide;

2. Colon et Covercrop (couverture);

3. Maiss et Velvet bean, puis Avoine d'hiver;

ou encore :

1. Arachide;

2. Maīs et Velvet bean;

3. Maĩs et Cowpea, puis Avoine ou Seigle d'hiver.

Dans l'Inde, l'arachide est égálement cultivée en assolement avec le cotonnier : on pratique particulièrement les assolements suivants :

1. Cotonnier, Arachide,

et surtout :

2. Cotonnier (2 ans), Sorgho, Arachide.

Canne à sucre. - L'association de la culture de la canne à sucre avec celle de fourrages a été utilisée en divers pays, notamment pour obtenir du fumier de ferme; la canne est utilisée comme fourrage en NouvellesGalles dư Sud, elle est même plus importante comme fourrage que comme plante à sucre, dans les fermes à bétail laitier; on y pratique une rotation comprenant maïs, puis sorgho, ensuite céréales d'hiver et finalement canne-fourrage:

Dans les Indes orientales néerlandaises, on pratique en rotation la culture de la canne à sucre, laquelle ne tient le terrain qu'un an sur trois, le reste étant consacré au maîs, à des légumineuses, à des cultures vivrières diverses.

Dans le Punjab, dans les sols alcalins, on cultive le riz, puis une rotation comprenant le trèfle d'Alexandrie, la canne à sucre, le coton.

\section{BIBLIOGRAPIIE}

1944. WhYTe (R.-0.). - Alternate husbandry (Trends in différént countries and régions). Imperial Bureaux of Pastures, Londres.

1944. Jacks (G.-V.). - The influence of herbage rotalions on the soil in a Alternate husbandry $n, \mathrm{p} .56$.

1940. KovalevsKaja (N:-P.): - The effect of a herbage ley in the physical and chemical properties of chesnut solonets soils. Pedology, $n^{\circ} 12$, p. 20.

1944. Paterson (D.-D.). - - Grassland management in tho West Indiea. In The Provision of animal fodder in tropical and sublropical countries, p. 7. - Imperial Bureau of Pastures, Londres. 